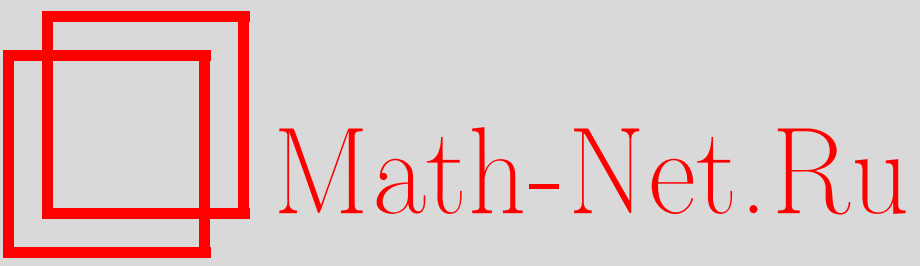

В. А. Горькавый, Конгруенции Бьянки двумерных поверхностей в $E^{4}$, Maтем. сб., 2005, том 196, номер 10, 79-102

DOI: https://doi.org/10.4213/sm1426

Использование Общероссийского математического портала Math-Net.Ru подразумевает, что вы прочитали и согласны с пользовательским соглашением

http://www. mathnet.ru/rus/agreement

Параметры загрузки:

IP : 54.197 .130 .99

26 апреля 2023 г., 14:57:11 
УДК 514

\title{
В.А. Горькавый
}

\section{Конгруенции Бьянки двумерных поверхностей в $E^{4}$}

\begin{abstract}
Исследуются псевдосферические конгруенции Бьянки в четырехмерном евклидовом пространстве $E^{4}$. Доказано, что фокалњные поверхности каждой такой конгруенции имеют постоянную отрицательную гауссову кривизну. Получены геометрическоеи аналитическое описания специальных псевдосферических поверхностей в $E^{4}$, допускающих конгруенцию Бьянки.

Библиография: 8 названий.
\end{abstract}

\section{Введение}

В настоящей статье изучаются двумерные поверхности с постоянной отрицательной гауссовой кривизной в четырехмерном евклидовом пространстве $E^{4}$. Теория псевдосферических поверхностей в трехмерном евклидовом пространстве $E^{3}$ и обобщающая ее теория $n$-мерных псевдосфрерических подмногообразий в $(2 n-1)$ мерном евклидовом пространстве являются хорошо изученньми направлениями классической дифференциальной геометрии, содержащими множество глубоких и красивых результатов (см., например, [1; гл. 10], [2]). Кроме того, теория псевдосферических поверхностей послужила становлению теории солитонов, в рамках которой получила затем свое дальнейшее развитие: уравнение sin-Гордона, описывающее поверхности с постоянной отрицательной гауссовой кривизной в $E^{3}$, и обобщающая его система нелинейных уравнений, описывающая псевдосферические подмногообразия $F^{n} \subset E^{2 n-1}$, представляют собой один из самых содержательных и наглядных примеров интегрируемых систем. Геометрически изящные конструкции преобразований псевдосферических поверхностей, найденные и изученные Л. Бьянки, А.В. Беклундом, Г. Дарбу (обобшенные на $n$-мерный случай в работах Ю.А. Аминова, см. [1; гл. 10, §24], К. Тенеблат и Ч.-Л. Тернг [3], [4]), стимулировали возникновение и развитие одного из важнейших объектов теории солитонов - понятия преобразования Бэклунда нелинейного уравнения.

В отличие от классической теории геометрия псевдосферических поверхностей в четырехмерном евклидовом пространстве $E^{4}$ представляет собой менее изученную область. Безусловно, имеется большое число работ, в которых такие поверхности исследуются, например, с точки зрения вопросов существования. Однако проблема вложимости этой теории в рамки теории солитонов остается пока что открытой. Не ясно, представляют ли псевдосферические поверхности $F^{2}$ в $E^{4}$ пример интегрируемой системы; не построена система дифференциальных уравнений,

Работа вьполненапри частичной поддержке Министерства образования и науки Украины. 
которая бы обобщала уравнение sin-Гордона и эффективно описьвала такие поверхности; не выяснен вопрос о существовании аналога преобразований Бэклунда. Первые результаты в данном направлении были получены Ю.А. Аминовым и А. Сымом в [5]. Было построено понятие преобразования Бьянки псевдосферических поверхностей в $E^{4}$, обобшающее классическое преобразование Бьянки для $F^{2}$ в $E^{3}[6]$ и аналогичное многомерному преобразованию Бьянки для $F^{n}$ в $E^{2 n-1}$ [1; гл. 10, $§ 24]$. А именно, пусть $F^{2} \subset E^{4}$ - поверхность постоянной отрицательной гауссовой кривизны $K=-1$, параметризованная орициклическими координатами $u, v$ так, что ее метрика имеет вид $d s^{2}=d u^{2}+e^{2 u} d v^{2}$. Пусть $\rho(u, v)-$ соответствующий радиус-вектор $F^{2}$. Преобразованием Бъянки поверхности $F^{2}$ называется регулярная поверхность $\widetilde{F}^{2}$ в $E^{4}$ с радиус-вектором $\widetilde{\rho}=\rho-\partial_{u} \rho$. Было показано, что в общем случае поверхность $\widetilde{F}^{2}$ уже не является псевдосферической. Далее было выяснено, в каком случае преобразование Бьянки переводит псевдосферическую поверхность в псевдосферическую. Соответствующий специальный класс псевдосферических поверхностей описывается системой нелинейных уравнений, установленной в [5]. Как следствие было показано, что псевдосферические поверхности с плоской нормальной связностью в $E^{4}$, не лежашие в гиперплоскостях $E^{3} \subset E^{4}$, не допускают преобразований Бьянки.

Мы предлагаем несколько иной подход к построению аналога преобразований Бьянки для поверхностей в $E^{4}$, основываясь на классических конструкциях Бьянки и Бэклунда. Пусть $F^{2}$ и $\widetilde{F}^{2}$ - регулярные двумерные поверхности в четырехмерном евклидовом пространстве $E^{4}$. Линейной конгруениией назьвают диффеоморфизм $\psi: F^{2} \rightarrow \widetilde{F}^{2}$, обладаюший свойством “двойного касания": для каждой точки $P \in F^{2}$ прямая линия, соединяющая $P$ с ее образом $\psi(P)=\widetilde{P} \in \widetilde{F}^{2}$, касается поверхностей $F^{2}$ и $\widetilde{F}^{2}$. Конгруенцией Бъянки будем называть линейную конгруенцию, обладающую двумя дополнительными свойствами:

$\left.\mathrm{B}_{1}\right)$ расстояние $l$ между соответствуюшими точками $P$ и $\psi(P)=\widetilde{P}$ постоянно и равно 1;

$\mathrm{B}_{2}$ ) угол между касательными плоскостями $T_{P} F^{2}$ и $T_{\widetilde{P}} \widetilde{F}^{2}$ в соответствующих точках равен $\pi / 2$.

Как было показано Ю.А. Аминовым и А. Сымом в [5], построенное ими преобразование Бьянки не является, вообше говоря, линейной конгруенцией. Однако если преобразование Бьянки псевдосферической поверхности в $E^{4}$ представляет собой линейную конгруенцию, то преобразованная поверхность тоже псевдосферична; кроме того, не составляет труда показать, что в этом случае само преобразование Бьянки обладает дополнительными свойствами $\left.\mathrm{B}_{1}\right), \mathrm{B}_{2}$ ). Таким образом, понятие конгруенции Бьянки в $E^{4}$ является не менее естественным, чем понятие преобразования Бьянки из [5]. Более того, в определении конгруенции Бьянки нет никаких априорных предположений о поверхностях $F^{2}$ и $\widetilde{F}^{2}$, а сами эти поверхности входят в рассматриваемую конструкцию на равных правах.

Основной целью статьи является доказательство того факта, что, как и в классических случаях, конгруениия Бъянки в $E^{4}$ связывает между собой только псевдосферические поверхности. Также изучается вопрос о том, насколько широко множество конгруенций Бьянки, допускаемых заданной псевдосферической 
поверхностью; дается эффективное описание тех псевдосферических поверхностей в $E^{4}$, которые допускают преобразования Бьянки; указана взаимосвязь геометрических свойств псевдосферических поверхностей, связанных конгруенцией Бьянки.

Чтобы исключить из рассмотрения гиперповерхности в гиперплоскостях $E^{3} \subset E^{4}$, являющихся хорошо изученным объектом классической теории, воспользуемся понятием точечной коразмерности (размерности первого нормального пространства) и будем всегда предполагать, что точечная коразмерность рассматриваемых поверхностей в $E^{4}$ равна 2 . Нам понадобится следующая классификация точек таких поверхностей:

1) гиперболические точки - в каждой из них в касательной плоскости поверхности имеются два однозначно определенных сопряженных направления;

2) параболические точки - в каждой из них в касательной плоскости поверхности имеется однозначно определенное асимптотическое направление;

3) эллиптические точки - в каждой из них в касательной плоскости поверхности нет ни асимптотических, ни сопряженных направлений.

Такая классификация соответствует известным классификациям, рассмотренным в $[1 ;$ гл. $8, \S 5]$ и [7]. Поверхность, состоящая из гиперболических точек, несет однозначно определенную регулярную сеть сопряженных линий - обычно она называется картановой поверхностью. Поверхность, которая состоит из параболических точек и которую мы будем называть $P$-поверхностью, однозначным образом расслаивается на асимптотические линии. Наконец, поверхности, которые состоят из эллиптических точек и которые мы будем называть $E$-поверхностямии, не содержат ни асимптотических, ни сопряженных линий. Следует напомнить, что произвольная поверхность в трехмерном евклидовом пространстве $E^{3}$ несет большое множество сетей сопряженных линий, поскольку для любого касательного направления на такой поверхности всегда существует сопряженное направление.

Важность сопряженных направлений и необходимость использования упомянутой классификации объясняется следующим классическим фактом, доказательство которого мы приводим для полноты изложения в $\S 1$ : прямые линии линейной конгруенции $\psi: F^{2} \rightarrow \widetilde{F}^{2}$ в $E^{4}$ задают направления на поверхностях $F^{2}$ и $\widetilde{F}^{2}$, для которых существуют сопряженные направления. Кроме того, по заданному полю направлений на поверхности $F^{2} \subset E^{4}$, для которого существует поле сопряженных направлений, в общем случае можно построить линейную конгруенцию $\psi: F^{2} \rightarrow \widetilde{F}^{2}$. Как следствие поверхности с точечной коразмерностью 2 в $E^{4}$ допускают более узкое множество линейных конгруенций, чем поверхности в $E^{3}$. В частности, $E$-поверхности вообще не допускают линейных конгруенций. Кроме того, можно показать, что $P$-поверхности, допускающие линейные конгруенции, это в точности линейчатые поверхности, а конгруенций Бьянки такие поверхности вообще не допускают.

Таким образом, мы сосредоточиваем основное внимание на картановых поверхностях в $E^{4}$. В общем случае каждая из картановых поверхностей допускает в точности две линейные конгруенции, соответствующие линейным конгруенциям сопряженных сетей, хорошо известным в классической терминологии как первое и минус первое преобразования Лапласа [2]. 
Основным результатом является доказательство следуюшего утверждения.

Теорема 1. Пусть $F^{2} \subset E^{4}$ - картанова поверхность, и пусть $\psi: F^{2} \rightarrow \widetilde{F}^{2}$ конгруенция Бвянки. Тогда гауссова кривизна $K$ поверхности $F^{2}$ постоянна и равна -1. При этом сеть сопряженных линий на $F^{2}$ нигде не ортогональна, а координатнье линии $v=$ const сопряженной сети, касательнье прямые которых представляют конгруенцию Бъянки, образуют семейство параллельных геодезических с орициклическими ортогональными траекториями.

Заметим, что вследствие симметрии рассматриваемой конструкции поверхность $\widetilde{F}^{2}$ обладает в обшем случае теми же свойствами, что и поверхность $F^{2}$. Таким образом, если две поверхности $F^{2}$ и $\widetilde{F}^{2}$ в $E^{4}$ связаны конгруенцией Бьянки, то они являются псевдосферическими поверхностями гаусовой кривизны $K=-1$, как и в классической трехмерной теории. С другой стороны, в отличие от классического случая в $E^{4}$ уже не любая псевдосферическая поверхность с точечной коразмерностью 2 допускает конгруенцию Бьянки: такая поверхность должна допускать введение двух систем координат, орициклической и сопряженной, имеющих одно общее семейство координатных линий. Орициклическая система является естественным объектом внутренней геометрии плоскости Лобачевского, тогда как требование наличия системы сопряженных координат (внешне-геометрический объект), как мы отметили выше, является сушественным ограничением для поверхностей в $E^{4}$. Например, нет никаких причин отрицать сушествование псевдосферических $E$ - или $P$-поверхностей в $E^{4}$, а такие поверхности не будут допускать конгруенций Бьянки.

В дополнение к теореме 1 мы доказываем, что найденные необходимые условия являются также и достаточными - аналогичное утверждение было сформулировано в иных терминах и доказано в [5].

ТеОрема 2. Пусть $F^{2} \subset E^{4}$ - картанова поверхность с гауссовой кривизной $K=-1$. Предположим, что на поверхности $F^{2}$ можсно ввести сопряхсенные координаты $u, v$ такие, что семейство координатных линий $v=$ const представлено параллельными геодезическими с ортогональными траекториями, т.е. метрика поверхности $F^{2}$ представляется в виде

$$
d s^{2}=(d \varphi(u, v))^{2}+e^{2 \varphi(u, v)}(d v)^{2}
$$

где $\varphi(u, v)$ - некоторая функиия с $\partial_{u} \varphi \neq 0$. Предположим, что сопряжсеннье координатьи $u, v$ нигде не ортогональны, т.е. $\partial_{v} \varphi \neq 0$. Тогда преобразование $F^{2} \rightarrow \widetilde{F}^{2}$, задаваемое формулой

$$
\widetilde{\rho}=\rho-\frac{1}{\partial_{u} \varphi} \partial_{u} \rho
$$

представляет конгруенщию Бьянки.

Следует подчеркнуть, что картанова псевдосферическая поверхность в $E^{4}$ допускает не более двух конгруенций Бьянки, что разительно отличает трехмерньй 
случай от четырехмерного. Интересно было бы построить псевдосферические картановы поверхности, допускаюшие в точности две конгруенции Бьянки. На наш взгляд, таких поверхностей не существует, и если уж поверхность допускает конгруенцию Бьянки, то такая конгруенция единственна. Эта дало бы повод использовать термин “пара псевдосферических картановых поверхностей в $E^{4}$, связанных преобразованием Бьянки”, по аналогии, например, с термином “пара изотермических поверхностей, связанных преобразованием Кристоффеля" .

Доказательство теорем 1 и 2 проведено в $\S \S 2,3$, где также установлены некоторые взаимосвязи геометрических свойств картановых поверхностей, связанных конгруенцией Бьянки. Мы находим эффективное представление для таких поверхностей в терминах решений специальной системы нелинейных уравнений: это позволяет убедиться в сушествовании описанных в теореме 2 псевдосферических поверхностей, с одной стороны, и выяснить, как преобразуются основные фундаментальные формы поверхностей под действием конгруенции Бьянки, с другой стороны (теоремы 3 и 4). В работе [5] был найден один из вариантов такого описания в терминах орициклических координат, мы же используем сопряженные координаты, более удобные и естественные в рассматриваемой задаче. Основной результат в этом направлении можно сформулировать следующим образом: nсевдосферическая картанова поверхность $F^{2}$ в $E^{4}$, несущая конгруенцию Бъянки $F^{2} \rightarrow \widetilde{F}^{2}$, описывается решением $\{\varphi(u, v), P(u, v), Q(u, v)\}$ системы дифференииальных уравнений

$$
\begin{gathered}
\partial_{u u}^{2} \varphi \cdot e^{2 \varphi}+2\left(\partial_{u} \varphi\right)^{2} e^{2 \varphi}-\partial_{v v}^{2} \varphi \cdot e^{-2 \varphi}+2\left(\partial_{v} \varphi\right)^{2} e^{-2 \varphi}+P Q+1=0, \\
\partial_{u} P-\partial_{u} \varphi Q e^{2 \varphi}=0, \\
\partial_{v} Q+\partial_{v} \varphi P e^{-2 \varphi}=0,
\end{gathered}
$$

удовлетворяющим условиям $\partial_{u} \varphi>0, \partial_{v} \varphi>0, P \neq 0$ и $Q \neq 0$. При этом самой конгруениии Бъянки отвечает следующее преобразование решений этой системьл:

$$
\{\varphi(u, v), P(u, v), Q(u, v)\} \mapsto\{-\varphi(-v,-u),-Q(-v,-u),-P(-v,-u)\} .
$$

В статье приводятся выражения фундаментальных форм поверхностей $F^{2}$ и $\widetilde{F}^{2}$ в терминах функций $\varphi(u, v), P(u, v), Q(u, v)$, сами же указанные уравнения представляют собой систему уравнений Гаусса-Кодацци-Риччи как для $F^{2}$, так и для $\widetilde{F}^{2}$. Выписанная система уравнений может рассматриваться как некий аналог

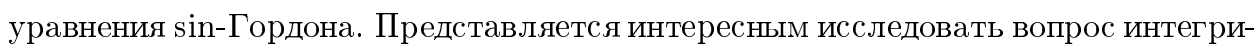
руемости этой системы, нахождение ее конкретных решений. В завершаюшем параграфе статьи находятся решения типа "бегущей волны"

$$
\{\varphi(a u+b v), P(a u+b v), Q(a u+b v)\}
$$

и изучается вопрос сушествования решений вида

$$
\{\varphi(u, v), P(\varphi(u, v)), Q(\varphi(u, v))\} .
$$

Автор выражает благодарность профессорам Ю. А. Аминову и А. Сьму за стимулируюшее и плодотворное обсуждение работы. 


\section{$\S 1$. Линейные конгруенции в $E^{4}$}

По определению линейной конгруенцией в $E^{4}$ называют локальньй диффеоморфизм регулярных поверхностей $\psi: F^{2} \rightarrow \widetilde{F}^{2}$ в $E^{4}$, обладающий свойством “двойного касания" : для каждой точки $P \in F^{2}$ прямая линия, соединяющая $P$ с ее образом $\psi(P)=\widetilde{P} \in \widetilde{F}^{2}$, касается поверхностей $F^{2}$ и $\widetilde{F}^{2}$. Часто линейная конгруенция ассоциируется с соответствующим двупараметрическим семейством прямых в $E^{4}$, а $F^{2}$ и $\widetilde{F}^{2}$ называются фокальными поверхностями конгруенции. В отличие от линейных конгруенций в $E^{3}$ линейные конгруенции в $E^{4}$ образуют весьма специальный класс двупараметрических семейств прямых. Прежде всего, мы рассмотрим следующий вопрос: когда поле касательных прямых, выбранное на заданной поверхности $F^{2}$, соответствует некоторой линейной конгруенции $F^{2} \rightarrow \widetilde{F}^{2}$ ?

Предположим, что поверхность $F^{2}$ задана радиус-вектором $r=\rho(u, v)$, и обозначим через $X$ поле направляющих векторов выбранного поля прямых линейной конгруенции. Векторное поле $X$ разлагается в линейную комбинацию базисных векторов: $X=X^{1} \partial_{u} \rho+X^{2} \partial_{v} \rho$. Радиус-вектор поверхности $\widetilde{F}^{2}$ должен записываться в виде

$$
\widetilde{\rho}(u, v)=\rho+\lambda\left(X^{1} \partial_{u} \rho+X^{2} \partial_{v} \rho\right) .
$$

Задача состоит в нахождении гладкой функции $\lambda(u, v)$ такой, чтобы $F^{2}$ и $\widetilde{F}^{2}$ обладали свойством двойного касания, т.е. чтобы векторное поле $X$ разлагалось в линейную комбинацию касательных векторов $\partial_{u} \widetilde{\rho}, \partial_{v} \widetilde{\rho}$ поверхности $\widetilde{F}^{2}$.

Дифференцируя (1) и находя $\partial_{u} \widetilde{\rho}, \partial_{v} \widetilde{\rho}$, получаем

$$
\begin{aligned}
\partial_{u} \widetilde{\rho}= & \partial_{u} \rho\left(1+\partial_{u}\left(\lambda X^{1}\right)+\lambda X^{1} \Gamma_{11}^{1}+\lambda X^{2} \Gamma_{12}^{1}\right) \\
& +\partial_{v} \rho\left(\partial_{u}\left(\lambda X^{2}\right)+\lambda X^{1} \Gamma_{11}^{2}+\lambda X^{2} \Gamma_{12}^{2}\right)+\sum_{\sigma=1}^{2} n_{\sigma}\left(X^{1} L_{11}^{\sigma}+X^{2} L_{12}^{\sigma}\right) \lambda \\
= & \partial_{u} \rho A_{1}+\partial_{v} \rho B_{1}+n_{1} C_{1}+n_{2} D_{1}, \\
\partial_{v} \widetilde{\rho}= & \partial_{u} \rho\left(\partial_{v}\left(\lambda X^{1}\right)+\lambda X^{1} \Gamma_{12}^{1}+\lambda X^{2} \Gamma_{22}^{1}\right) \\
& +\partial_{v} \rho\left(1+\partial_{v}\left(\lambda X^{2}\right)+\lambda X^{1} \Gamma_{12}^{2}+\lambda X^{2} \Gamma_{22}^{2}\right)+\sum_{\sigma=1}^{2} n_{\sigma}\left(X^{1} L_{12}^{\sigma}+X^{2} L_{22}^{\sigma}\right) \lambda \\
= & \partial_{u} \rho A_{2}+\partial_{v} \rho B_{2}+n_{1} C_{2}+n_{2} D_{2},
\end{aligned}
$$

здесь и ниже через $g_{i j}$ и $\Gamma_{i j}^{k}$ обозначены коэффициенты метрики и символы Кристоффеля поверхности $F^{2}$, через $n_{1}, n_{2}$ обозначены поля нормалей к $F^{2}$, образующих в каждой точке $P \in F^{2}$ ортонормированный базис в нормальной плоскости $N_{P} F^{2}$, а $L_{i j}^{1}$ и $L_{i j}^{2}$ обозначают коэффициенты вторых квадратичных форм $F^{2}$ относительно $n_{1}$ и $n_{2}$. Записывая разложение векторов $\partial_{u} \widetilde{\rho}, \partial_{v} \widetilde{\rho}$ и $X$ в базисе $\partial_{u} \rho$, $\partial_{v} \rho, n_{1}, n_{2}$ в $E^{4}$, легко увидеть, что условие двойного касания эквивалентно равенству

$$
\operatorname{Rank}\left(\begin{array}{cccc}
A_{1} & B_{1} & C_{1} & D_{1} \\
A_{2} & B_{2} & C_{2} & D_{2} \\
X^{1} & X^{2} & 0 & 0
\end{array}\right)=2
$$


причем первая и вторая строки выписанной матрицы должны быть линейно независимы, чтобы гарантировать регулярность поверхности $\widetilde{F}^{2}$. Условие (4) представляет собой систему алгебраических и дифференциальных уравнений для нахождения функции $\lambda(u, v)$. Ясно, что $\lambda \equiv 0$ удовлетворяет (4), так как $X$ образовано касательньми векторами к самой исходной поверхности $F^{2}$. Нас же будет интересовать не обрашаюшееся в нуль решение $\lambda(u, v)$ системы $(4)$, а линейную конгруенцию в таком случае будем называть нетривиальной.

Поскольку $X^{1}$ и $X^{2}$ не равны нулю одновременно, из (4) следует, что $C_{1} D_{2}-C_{2} D_{1}=0$. Поэтому если сушествует не обращающееся в нуль решение $\lambda$ системы (4), то

$$
\left(X^{1}\right)^{2}\left|\begin{array}{ll}
L_{11}^{1} & L_{11}^{2} \\
L_{12}^{1} & L_{12}^{2}
\end{array}\right|+X^{1} X^{2}\left|\begin{array}{cc}
L_{11}^{1} & L_{11}^{2} \\
L_{22}^{1} & L_{22}^{2}
\end{array}\right|+\left(X^{2}\right)^{2}\left|\begin{array}{cc}
L_{12}^{1} & L_{12}^{2} \\
L_{22}^{1} & L_{22}^{2}
\end{array}\right|=0
$$

Как следствие касательное векторное поле $X$ на $F^{2}$ не может быть выбрано произвольньм, оно обязано удовлетворять условию (5). Это однородное уравнение второго порядка, чьи коэффициенты определяются вторьми квадратичными формами $F^{2}$. Такое уравнение в точности описьвает сопряженные направления на $F^{2}$.

Напомним, что направления $\left(V^{1}: V^{2}\right),\left(W^{1}: W^{2}\right)$ в касательной плоскости $T_{P} F^{2}$ сопряжены друг другу, если $L_{i j}^{1} V^{i} W^{j}=0, L_{i j}^{2} V^{i} W^{j}=0$. Самосопряженное направление назьвается асимптотическим, если координаты удовлетворяют условиям $L_{i j}^{1} V^{i} V^{j}=0, L_{i j}^{2} V^{i} V^{j}=0$. Условие сопряженности можно переписать как систему линейных уравнений для $W^{1}, W^{2}$ :

$$
\left(\begin{array}{ll}
L_{11}^{1} V^{1}+L_{12}^{1} V^{2} & L_{12}^{1} V^{1}+L_{22}^{1} V^{2} \\
L_{11}^{2} V^{1}+L_{12}^{2} V^{2} & L_{12}^{2} V^{1}+L_{22}^{2} V^{2}
\end{array}\right)\left(\begin{array}{l}
W^{1} \\
W^{2}
\end{array}\right)=\left(\begin{array}{c}
0 \\
0
\end{array}\right)
$$

Система имеет ненулевое решение тогда и только тогда, когда $V^{1}, V^{2}$ являются решением однородного уравнения второго порядка

$$
\left(V^{1}\right)^{2}\left|\begin{array}{cc}
L_{11}^{1} & L_{11}^{2} \\
L_{12}^{1} & L_{12}^{2}
\end{array}\right|+V^{1} V^{2}\left|\begin{array}{cc}
L_{11}^{1} & L_{11}^{2} \\
L_{22}^{1} & L_{22}^{2}
\end{array}\right|+\left(V^{2}\right)^{2}\left|\begin{array}{cc}
L_{12}^{1} & L_{12}^{2} \\
L_{22}^{1} & L_{22}^{2}
\end{array}\right|=0
$$

Ясно, что $W^{1}, W^{2}$ тоже должны быть решением этого уравнения. Сравнивая (5) и (6), получаем следующее условие на $X$, необходимое для существования линейной конгруенции.

Лемма 1. Пусть $\psi: F^{2} \rightarrow \widetilde{F}^{2}$ - нетривиальная линейная конгруенция. Тогда прямые линейной конгруенции $\psi$ представляют поле касательных направленийна $F^{2}$, для которого существует поле сопряженных направлений.

Очевидно ввиду симметричности рассматриваемой конструкции, что прямые линейной конгруенции $\psi$ представляют поле касательных направлений, для которого существует поле сопряженных направлений, и на поверхности $\widetilde{F}^{2}$. Позже мы покажем, что найденное условие является не только необходимым, но и достаточным в общем случае для существования линейной конгруенции. 
Непосредственњым следствием леммы 1 является существенное различие в поведении линейных конгруенций в $E^{3}$ и в $E^{4}$. Анализируя условие сопряженности, аналитически выраженное уравнением (6), легко увидеть, что разрешимость (6) зависит от коэффициентов вторых квадратичных форм и в первую очередь - от точечной коразмерности $F^{2}$. Напомним, что точечной коразмерностью (размерностью первого нормального пространства) поверхности $F^{2}$ называют величину

$$
\operatorname{codim}_{P}=\operatorname{Rank}\left(\begin{array}{lll}
L_{11}^{1} & L_{12}^{1} & L_{22}^{1} \\
L_{11}^{2} & L_{12}^{2} & L_{22}^{2}
\end{array}\right),
$$

зависящую от точки $P \in F^{2}$. Если $\operatorname{codim}_{P}$ равна 2 , то (6) является невырожденным однородным уравнением второго порядка. В этом случае в зависимости от точки $P \in F^{2}$ уравнение (6) имеет либо два линейно независимых решения $\left(V^{1}: V^{2}\right),\left(W^{1}: W^{2}\right)$, либо одно ненулевое решение $\left(V^{1}: V^{2}\right)$, либо не имеет решений. Соответственно в касательной плоскости $T_{P} F^{2}$ будет либо пара однозначно определенных сопряженных направлений, либо одно однозначно определенное асимптотическое направление, либо не будет ни сопряженных, ни асимптотических направлений. Если $\operatorname{codim}_{P}$ равна 1 в точке $P$, то уравнение (6) вырождается в тождество и, значит, для любого направления $V$ в $T_{P} F^{2}$ будет существовать однозначно определенное сопряженное направление $W$; такая ситуация возникает во всех точках, например когда $F^{2}$ принадлежит некоторой аффинной гиперплоскости $E^{3} \subset E^{4}$. Наконец, если $\operatorname{codim}_{P}$ равна 0 в точке $P$, то все направления в $T_{P} F^{2}$ сопряжены друг другу. Таким образом, мы видим, что понятие сопряженности для векторов на поверхности в $E^{4}$, а значит, и понятие линейной конгруенции в $E^{4}$, связано со значительно более существенными ограничениями, чем в классической трехмерной ситуации.

Проведенный анализ уравнения (6), описывающего сопряженные направления, позволяет сформулировать

УТВЕРЖ ДЕНИЕ 1. Предположим, что точечная коразмерность поверхности $F^{2} \subset E^{4}$ в точке $P$ равна 2. Тогда имеет место одна и только одна ситуация из трех:

1) касательная плоскость $T_{P} F^{2}$ содержит единственную пару линейно независимых сопряженных направлений $V, W$, m.e. $L(V, W)=0$;

2) касательная плоскость $T_{P} F^{2}$ содержит единственное асимптотическое направление $V$, т.е. $L(V, V)=0$;

3) в касательной плоскости $T_{P} F^{2}$ нет ни сопряженных, ни асимптотических направлений, но зато для любого вектора $V$ существует единственный $\pm W$ (комплексно-сопряженный $\kappa V)$ такой, что $L(V+i W, V-i W)=0$.

В зависимости от того, какая из трех ситуаций имеет место, точка $P$ называется гиперболической, параболической или эллиптической соответственно. Эта классификация эквивалентна классификации точек поверхности $F^{2}$ в $E^{4}$ с помощью грассманова образа, предложенной Аминовьм, и аффинной классификации, исследованной С.З. Шефелем, А. А. Борисенко и др., см. [1; гл. 8, § 5], [7], [8]. В частности, Аминов рассмотрел секционную кривизну $\bar{K}$ многообразия Грассмана $G_{2,4}$ по 
двумерньм плошадкам, касательным к грассманову образу поверхности $F^{2} \subset E^{4}$. Кривизна $\bar{K}$ принимает следующие значения: $0<\bar{K}<1$, если $P$ гиперболическая; $\bar{K}=1$, если $P$ параболическая; и $1<\bar{K}<2$, если $P$ эллиптическая.

Поверхность в $E^{4}$, состоящая из гиперболических точек, представляет собой картанову поверхность, такая поверхность характеризуется наличием однозначно определенной регулярной сети сопряженных линий. Поверхность в $E^{4}$, все точки которой параболические, мы будем называть $P$-поверхностью, такая поверхность однозначньм образом расслаивается на асимптотические линии. Поверхность в $E^{4}$, все точки которой эллиптические, мы будем называть $E$-поверхностью, такая поверхность не содержит ни асимптотических, ни сопряженных направлений. Напомним, что произвольная поверхность в $E^{3}$ несет большое множество сетей сопряженных линий, в этом смысле трехмерный случай существенно отличается от четырехмерного.

Отметим тот очевидньй факт, что с локальной точки зрения картановы и $E$-поверхности представляют собой ситуацию общего положения, тогда как $P$-поверхности и поверхности с точечной коразмерностью меньше 2 (поверхности в гиперплоскостях $E^{3} \subset E^{4}$ и поверхности с вырожденным грассмановым образом) представляют собой более частные, специальные классы поверхностей в $E^{4}$.

Принимая во внимание описанную классификацию и доказанную выше лемму 1 , получаем следующее утверждение.

ЛЕмма 2. Пусть $F^{2} \subset E^{4}$ - регулярная поверхность с постоянной точечной коразмерностью 2. Пусть $\psi: F^{2} \rightarrow \widetilde{F}^{2}$ - нетривиальная линейная конгруенция. Тогда $F^{2}$ состоит из гиперболических и параболических точек. При этом поле прямых линейной конгруениии $\psi$ обладает следующим свойством: в каждой гиперболической точке на $F^{2}$ прямая конгруенции представляет одно из двух сопряженных направлений в касательной плоскости, а в каждой из параболических точек - асимптотическое направление.

СЛЕДСТВИЕ. Произвольная картанова поверхность в $E^{4}$ допускает не более двух нетривиальных линейных конгруенций. Произвольная $P$-поверхность в $E^{4}$ допускает не более одной нетривиальной линейной конгруенции. Произвольная Е-поверхность в $E^{4}$ не допускает нетривиальных линейных конгруенций.

Подводя итог, с локальной точки зрения мы можем дать следующую классификацию поверхностей в $E^{4}$, возможно допускающих линейные конгруенции: картановы поверхности, $P$-поверхности, поверхности с вырожденным грассмановым образом, поверхности в аффинных гиперплоскостях $E^{3} \subset E^{4}$. Далее внимание в основном будет уделено картановьм поверхностям, представляющим в данной ситуации наиболее общий класс поверхностей.

\section{§2. Линейные конгруенции картановых поверхностей}

Будем предполагать, что поверхность $F^{2} \subset E^{4}$ картанова. Она имеет точечную коразмерность 2 и состоит из гиперболических точек. В каждой точке на $F^{2}$ в касательной плоскости имеется единственная пара сопряженных направлений. 
Мы можем ввести локальные координаты $u, v$ на $F^{2}$ так, чтобы координатные линии касались сопряженных направлений и, таким образом, образовывали бы сеть сопряженных линий на $F^{2}$. Очевидно, что сеть сопряженных линий на картановой поверхности $F^{2}$ определена однозначно, а сами координаты допускают шкалируюшее преобразование $\widehat{u}=\widehat{u}(u), \widehat{v}=\widehat{v}(v)$. В данной параметризации обе вторые квадратичные формы диагональны: $L_{12}^{1}=0, L_{12}^{2}=0$. Кроме того, $L_{11}^{1} L_{22}^{2}-L_{22}^{1} L_{11}^{2} \neq 0$, поскольку точечная коразмерность поверхности $F^{2}$ равна 2 . (Если бы нормальная связность $F^{2}$ была плоской, то можно было бы диагонализовать и первую квадратичную форму, $g_{12}=0$, и тогда координатные кривые были бы линиями кривизны.)

Найдем аналитическое представление рассматриваемой линейной конгруенции $\psi: F^{2} \rightarrow \widetilde{F}^{2}$. Вследствие леммы 2 поле прямых линейной конгруенции $\psi$ должно сооответствовать одному из двух полей сопряженных направлений на $F^{2}$. Иначе говоря, в качестве направляющего векторного поля $X$ мы должны взять либо $\partial_{u} \rho$, либо $\partial_{v} \rho$. Не уменьшая общности, положим $X=\partial_{u} \rho$, т.е. $X^{1}=1, X^{2}=0$. Тогда условие двойного касания (4) примет вид:

$$
\operatorname{Rank}\left(\begin{array}{cccc}
1+\partial_{u} \lambda+\lambda \Gamma_{11}^{1} & \lambda \Gamma_{11}^{2} & \lambda L_{11}^{1} & \lambda L_{11}^{2} \\
\partial_{v} \lambda+\lambda \Gamma_{12}^{1} & 1+\lambda \Gamma_{12}^{2} & 0 & 0 \\
1 & 0 & 0 & 0
\end{array}\right)=2
$$

Так как $L_{11}^{1}$ и $L_{11}^{2}$ не могут быть равными нулю одновременно ввиду того, что $\operatorname{codim}_{P} \equiv 2$, условие (7) будет выполнено тогда и только тогда, когда

$$
1+\lambda \Gamma_{12}^{2}=0
$$

Таким образом, на векторное поле $X$ не налагается более никаких ограничений, и мы имеем алгебраическое уравнение для $\lambda$. Если $\Gamma_{12}^{2}$ не обрашается в нуль, то решением будет функция $\lambda=-1 / \Gamma_{12}^{2}$. Следовательно, для радиус-вектора поверхности $\widetilde{F}^{2}$ получаем выражение [2]

$$
\widetilde{\rho}=\rho-\frac{1}{\Gamma_{12}^{2}} \partial_{u} \rho
$$

Используя (9), установим некоторые геометрические свойства поверхности $\widetilde{F}^{2}$. Продифференцируем $\widetilde{\rho}$ и найдем касательные векторы $\widetilde{F}^{2}$ :

$$
\begin{gathered}
\partial_{u} \tilde{\rho}=\partial_{u} \rho\left(1-\partial_{u}\left(\frac{1}{\Gamma_{12}^{2}}\right)-\frac{1}{\Gamma_{12}^{2}} \Gamma_{11}^{1}\right)-\left(\partial_{v} \rho \Gamma_{11}^{2}+n_{\sigma} L_{11}^{\sigma}\right) \frac{1}{\Gamma_{12}^{2}} \\
\partial_{v} \widetilde{\rho}=-\partial_{u} \rho\left(\partial_{v}\left(\frac{1}{\Gamma_{12}^{2}}\right)+\frac{1}{\Gamma_{12}^{2}} \Gamma_{12}^{1}\right) .
\end{gathered}
$$

Как следствие легко видеть, что $\widetilde{F}^{2}$ регулярна тогда и только тогда, когда

$$
\widehat{A}=-\left(\partial_{v}\left(\frac{1}{\Gamma_{12}^{2}}\right)+\frac{1}{\Gamma_{12}^{2}} \Gamma_{12}^{1}\right)=\frac{1}{\left(\Gamma_{12}^{2}\right)^{2}}\left(\partial_{v} \Gamma_{12}^{2}-\Gamma_{12}^{2} \Gamma_{12}^{1}\right) \neq 0 .
$$


Далее, дифференцируя (10.2), получаем

$$
\partial_{u} \partial_{v} \widetilde{\rho}=\partial_{u} \rho\left(\partial_{u} \widehat{A}+\widehat{A} \Gamma_{11}^{1}\right)+\left(\partial_{v} \rho \Gamma_{11}^{2}+n_{\sigma} L_{11}^{\sigma}\right) \widehat{A}
$$

Не составляет труда убедиться в том, что $\partial_{u} \partial_{v} \widetilde{\rho}$ разлагается в линейную комбинацию векторов $\partial_{u} \widetilde{\rho}$ и $\partial_{v} \widetilde{\rho}$, касательных к $\widetilde{F}^{2}$. Это означает, что координатные $u$ - и $v$-кривые на $\widetilde{F}^{2}$ являются сопряженными. Таким образом, как и ожидалось ввиду симметричности рассматриваемой конструкции, сеть сопряженных линий на $F^{2}$ отображается линейной конгруенцией $\psi$ на сеть сопряженных линий на $\widetilde{F}^{2}$, т.е. $\psi$ может рассматриваться как линейная конгруенция сопряженных сетей [2].

УТВЕРЖДЕНИЕ 2. Пусть $F^{2} \subset E^{4}$ - картанова поверхность, параметризованная сопряженными координатами, $r=\rho(u, v)$ - ее радиус-вектор. Пусть $\psi: F^{2} \rightarrow \widetilde{F}^{2}$ - нетривиальная линейная конгруенция. Тогда $\psi$ представляется (с точностью до переобозначения $и \leftrightarrow v$ ) в виде

$$
\widetilde{\rho}=\rho-\frac{1}{\Gamma_{12}^{2}} \partial_{u} \rho
$$

и при этом имеет место (11). Линейная конгруениия $\psi$ сохраняет сопряженность координатных кривых.

Очевидно, верен и обратный факт.

УТВЕРЖДЕНИЕ 3. Пусть $F^{2} \subset E^{4}$ - картанова поверхность, параметризованная сопряженными координатами, $r=\rho(u, v)$ - ее радиус-вектор. Предположим, что $\Gamma_{12}^{2} \neq 0, \partial_{v} \Gamma_{12}^{2}-\Gamma_{12}^{2} \Gamma_{12}^{1} \neq 0$. Тогда радиус-вектор

$$
\widetilde{\rho}=\rho-\frac{1}{\Gamma_{12}^{2}} \partial_{u} \rho
$$

задает линейную конгруенцию.

Отметим, что выражение $\left(1 / \Gamma_{12}^{2}\right) \partial_{u} \rho$ инвариантно при любом шкалирующем преобразовании сопряженных координат $\widehat{u}=\widehat{u}(u), \widehat{v}=\widehat{v}(v)$. Как следствие, если поверхность $F^{2}$ и поле сопряженных направлений на $F^{2}$ заданы, то по ним однозначно восстанавливается линейная конгруенция $\psi$ и поверхность $\widetilde{F}^{2}$. Согласно классической терминологии поверхность $\widetilde{F}^{2}$ - первое преобразование Лапласа поверхности $F^{2}[2]$.

Аналогичньм образом можно проанализировать ситуацию, когда $X$ направлено вдоль второго поля сопряженных направлений $\partial_{v} \rho$ на $F^{2}$, т.е. $X^{1}=0, X^{2}=1$. Радиус-вектор поверхности $\widetilde{F}^{2}$ будет иметь в этом случае вид

$$
\widetilde{\rho}=\rho-\frac{1}{\Gamma_{12}^{1}} \partial_{v} \rho
$$

Поверхность $\widetilde{F}^{2}$ называется минус первым преобразованием Лапласа поверхности $F^{2}[2]$.

Подводя итог, можем сделать следующий вывод: в противоположность классической трехмерной ситуации произвольная картанова поверхность $F^{2}$ в $E^{4}$ допускает в общем случае две и только две нетривиальные линейные конгруенции. 
Эти конгруенции, первое и минус первое преобразования Лапласа, определяются однозначно и соответствуют двум однозначно определенным полям сопряженных направлений на $F^{2}[2]$.

ЗАмЕчАниЕ. Было бы желательньм, чтобы линейная конгруенция преобразовывала картанову поверхность снова в картанову. Это свойство, очевидно, имеет место при дополнительном предположении, что точечная коразмерность преобразованной поверхности $\widetilde{F}^{2}$ равна 2 . Если же не делать этого предположения, то можно, вообще говоря, столкнуться с ситуацией, когда в некоторых точках на $\widetilde{F}^{2}$ точечная коразмерность становится меньше 2 , а значит, $\widetilde{F}^{2}$ уже не будет картановой.

\section{§3. Конгруенции Бьянки картановых поверхностей}

Теперь будем предполагать, что линейная конгруенция $\psi: F^{2} \rightarrow \widetilde{F}^{2}$ в $E^{4}$ обладает двумя дополнительными свойствами:

$\mathrm{B}_{1}$ ) расстояние $l$ между соответствующими точками $P \in F^{2}$ и $\psi(P)=\widetilde{P} \in \widetilde{F}^{2}$ постоянно и равно 1 , т.е. $l=|P \widetilde{P}| \equiv 1$;

$\mathrm{B}_{2}$ ) угол $\omega$ между касательньми плоскостями поверхностей $F^{2}$ и $\widetilde{F}^{2}$ в соответствующих по конгруенции точках постоянен и равен $\pi / 2$, т.е.

$$
\angle\left(T_{P} F^{2}, T_{\widetilde{P}} \widetilde{F}^{2}\right) \equiv \frac{\pi}{2}
$$

Естественно назвать такую линейную конгруенцию $\psi: F^{2} \rightarrow \widetilde{F}^{2}$ в $E^{4}$ конгруенцией Бьянки, подобно аналогичной классической конструкции в $E^{3}$. Очевидно, что поверхности $F^{2}$ и $\widetilde{F}^{2}$, связанные конгруенцией Бьянки, должны обладать некоторьми специальными свойствами, являющимися следствием выполения условий $\left.\mathrm{B}_{1}\right)$ и $\left.\mathrm{B}_{2}\right)$.

Мы по-прежнему будем рассматривать наиболее обший с локальной точки зрения случай и будем предполагать, что $F^{2} \subset E^{4}$ является картановой поверхностью. Воспользуемся параметризацией $r=\rho(u, v)$ поверхности $F^{2}$ с помощью сопряженных координат, рассмотренной в предыдущем параграфе. Как было установлено, линейная конгруенция $\psi$ описывается либо формулой (9), либо формулой (12). Не уменьшая общности, будем предполагать, что поверхность $\widetilde{F}^{2}$ задана радиус-вектором (9), при этом выполнено условие регулярности (11). Поскольку $\Gamma_{12}^{2}$ не обращается в нуль, мы можем, не уменьшая общности, специализировать выбор параметра $u$ таким образом, чтобы $\Gamma_{12}^{2}$ было положительным.

Расстояние $l$ между соответствующими точками на $F^{2}$ и $\widetilde{F}^{2}$ находится легко с помощью (9):

$$
l=\frac{\sqrt{g_{11}}}{\Gamma_{12}^{2}} .
$$

Найдем теперь угол $\omega$ между касательными плоскостями $T_{P} F^{2}$ и $T_{\widetilde{P}} \widetilde{F}^{2}$. Напомним, что взаимное расположение двух векторных подпространств $E_{1}^{2}, E_{2}^{2}$ в $E^{4}$ корректно определяется двумя углами [8]. Если пересечение $\zeta=E_{1}^{2} \cup E_{2}^{2}$ является прямой, то один из этих двух углов равен нулю, а второй определяется как 
угол между прямьми линиями в $E_{1}^{2}, E_{2}^{2}$, ортогональными прямой пересечения $\zeta$. Именно этот угол подразумевается в условии $\left.\mathrm{B}_{2}\right)$.

Вектор $\xi$ в $T_{P} F^{2}$, ортогональный $\partial_{u} \rho$, и вектор $\eta$ в $T_{\widetilde{P}} \widetilde{F}^{2}$, ортогональный $\partial_{v} \widetilde{\rho}=$ $\widehat{A} \partial_{u} \rho$, находятся элементарно:

$$
\begin{aligned}
& \xi=-g_{12} \partial_{u} \rho+g_{11} \partial_{v} \rho \\
& \eta=\Gamma_{11}^{2}\left(-g_{12} \partial_{u} \rho+g_{11} \partial_{v} \rho\right)+g_{11} L_{11}^{\sigma} n_{\sigma} .
\end{aligned}
$$

Как следствие для угла между $\xi$ и $\eta$, который как раз и равен углу $\omega$ между касательными плоскостями $T_{P} F^{2}$ и $T_{\widetilde{P}} \widetilde{F}^{2}$, получаем следуюшую формулу:

$$
\cos \omega=\frac{\Gamma_{11}^{2}}{\sqrt{\left(\Gamma_{11}^{2}\right)^{2}+\frac{g_{11}}{g_{11} g_{22}-g_{12}^{2}} \sum_{\sigma}\left(L_{11}^{\sigma}\right)^{2}}}
$$

ЗАмечАниЕ. Выражения в правых частях формул (13) и (14) для $l$ и $\cos \omega$ инвариантны относительно шкалирующих замен кординат $\widehat{u}=\widehat{u}(u), \widehat{v}=\widehat{v}(v)$, сохраняюших сопряженность. Их геометрическая интерпретация будет дана в приложении.

Таким образом, условия $\mathrm{B}_{1}$ ) и $\mathrm{B}_{2}$ ) можно записать в форме двух следующих равенств:

$$
\begin{aligned}
& \Gamma_{12}^{2}=\sqrt{g_{11}}, \\
& \Gamma_{11}^{2} \equiv 0 .
\end{aligned}
$$

Эти равенства содержат только коэффициенты метрики и символы Кристоффеля поверхности $F^{2}$, поэтому они представляют собой некоторые ограничения на внутреннюю геометрию $F^{2}$, дополняющие внешне-геометрическую сопряженность используемых координат на $F^{2}$.

ЛЕмма 3. Условия $\Gamma_{12}^{2}=\sqrt{g_{11}}>0$ и $\Gamma_{11}^{2} \equiv 0$ выполнены тогда и только тогда, когда существуют функиия $\varphi(u, v)$ с положительной производной $\partial_{u} \varphi$ и положстельная функция $f(v)$ такие, что

$$
g_{11}=\left(\partial_{u} \varphi\right)^{2}, \quad g_{12}=\partial_{u} \varphi \partial_{v} \varphi, \quad g_{22}=\left(\partial_{v} \varphi\right)^{2}+e^{2 \varphi} f^{2} .
$$

ДокАЗАТЕЛЬСтво. Запишем условие $\Gamma_{11}^{2} \equiv 0$ через коэффициенты $g_{i j}$ первой квадратичной формы:

$$
g_{11} \cdot \partial_{u} g_{12}-\frac{1}{2} g_{11} \cdot \partial_{v} g_{11}-\frac{1}{2} g_{12} \cdot \partial_{u} g_{11}=0 .
$$

Перепишем это равенство следуюшим образом:

$$
\partial_{u} \frac{g_{12}}{\sqrt{g_{11}}}=\partial_{v} \sqrt{g_{11}} .
$$


Как следствие существует функция $\varphi(u, v)$ такая, что

$$
\sqrt{g_{11}}=\partial_{u} \varphi \quad \text { и } \quad \frac{g_{12}}{\sqrt{g_{11}}}=\partial_{v} \varphi
$$

т.e.

$$
g_{11}=\left(\partial_{u} \varphi\right)^{2}, \quad g_{12}=\partial_{u} \varphi \partial_{v} \varphi
$$

при этом, очевидно, $\partial_{u} \varphi>0$.

Теперь проанализируем условие $\Gamma_{12}^{2}=\sqrt{g_{11}}$. С помощью коэффициентов $g_{i j}$ это равенство записывается в виде

$$
-\frac{1}{2} g_{12} \cdot \partial_{v} g_{11}+\frac{1}{2} g_{11} \cdot \partial_{u} g_{22}=\left(g_{11} g_{22}-g_{12}^{2}\right) \sqrt{g_{11}}
$$

Применяя (17), получаем

$$
\frac{1}{2} \partial_{u} g_{22}-\partial_{u} \partial_{v} \varphi \partial_{v} \varphi=\left(g_{22}-\left(\partial_{v} \varphi\right)^{2}\right) \partial_{u} \varphi
$$

Перепишем несколько иначе:

$$
\partial_{u}\left(g_{22}-\left(\partial_{v} \varphi\right)^{2}\right)=2\left(g_{22}-\left(\partial_{v} \varphi\right)^{2}\right) \partial_{u} \varphi
$$

Очевидно, полученное равенство имеет место тогда и только тогда, когда сушествует функция $f(v)$ такая, что

$$
g_{22}=\left(\partial_{v} \varphi\right)^{2}+e^{2 \varphi} f^{2}(v)
$$

Поскольку $g_{11} g_{22}-\left(g_{12}\right)^{2}=\left(\partial_{u} \varphi\right)^{2} e^{2 \varphi} f^{2}(v)>0$, функцию $f(v)$ можно считать положительной. Подчеркнем, что функция $\varphi(u, v)$ определяется однозначно с точностью до постоянного слагаемого, после чего функция $f(v)$ тоже определяется единственым образом.

Обратно, если существуют функция $\varphi(u, v)$ с положительной производной $\partial_{u} \varphi>0$ и положительная функция $f(v)$ такие, что $g_{11}=\left(\partial_{u} \varphi\right)^{2}, g_{12}=\partial_{u} \varphi \partial_{v} \varphi$, $g_{22}=\left(\partial_{v} \varphi\right)^{2}+e^{2 \varphi} f^{2}$, то будут выполнены условия (15) и (16) - в этом легко убедиться, вьчислив символь Кристоффеля $\Gamma_{12}^{2}$ и $\Gamma_{22}^{1}$. Лемма доказана.

Непосредственным следствием леммы 3 является следуюшее утверждение.

Теорема 1. Пусть $F^{2} \subset E^{4}-$ картанова поверхность, и пусть $\psi: F^{2} \rightarrow \widetilde{F}^{2}$ - конгруениия Бвянки. Тогда гауссова кривизна $K$ поверхности $F^{2}$ постоянна и равна -1. При этом сеть сопряжсенных линий на $F^{2}$ нигде не ортогональна, а координатные линии $v=$ const этой сети, касательные прямые которых представляют конгруенцию Бъянки, образуют семейство параллельньи геодезических с орициклическими ортогональными траекториями. 
ДокАЗАТЕЛЬСтво. Вследствие леммы 3 метрика поверхности $F^{2}$ приводится к виду $d s^{2}=(d \varphi)^{2}+e^{2 \varphi}(f d v)^{2}$. После замены координат $d \bar{u}=d \varphi(u, v)$, $d \bar{v}=f(v) d v$ мы получаем стандартный вид метрики плоскости Лобачевского в орициклических координатах $d s^{2}=(d \bar{u})^{2}+e^{2 \bar{u}}(d \bar{v})^{2}$. Поэтому $F^{2}$, очевидно, является псевдосферической поверхностью с постоянной гауссовой кривизной $K \equiv-1$. Напомним, что в орициклической системе координат координатные линии $\bar{v}=$ const - это параллельные геодезические, а ортогональные им координатные линии $\bar{u}=\mathrm{const}-$ орициклы. С другой стороны, семейство линий $v=\mathrm{const}$ представляется теми же кривыми, что и семейство координатных линий $\bar{v}=$ const.

Наконец, поскольку в рассматриваемой ситуации $\Gamma_{12}^{2}=\sqrt{g_{11}}=\left(\partial_{u} \varphi\right)^{2}$ и $\partial_{v} \Gamma_{12}^{2}-\Gamma_{12}^{1} \Gamma_{12}^{2}=\partial_{u} \varphi \partial_{v} \varphi=g_{12}$, что проверяется непосредственным вычислением, то условие (11), являющееся необходимым для существования конгруенции Бьянки и эквивалентное регулярности преобразованной поверхности $\widetilde{F}^{2}$, будет выполнено тогда и только тогда, когда $g_{12}=\partial_{u} \varphi \partial_{v} \varphi$ не обращается в нуль. Это и означает, что сеть сопряженных линий на $F^{2}$ нигде не ортогональна. Теорема доказана.

СлЕДСТВИЕ. Пусть $F^{2} \subset E^{4}$ - картанова поверхность, и пусть $F^{2} \rightarrow \widetilde{F}^{2}$ - конгруенция Бъянки. Тогда нормальная связность $F^{2}$ не может быть плоской.

ДокАЗАТЕльСтво. Если бы нормальная связность $F^{2}$ была плоской, то на $F^{2}$ можно было бы ввести координаты кривизны. Сеть сопряженных координатных линий на $F^{2}$ была бы образована линиями кривизны, а значит, была бы ортогональной, что противоречит необходимому условию $g_{12} \neq 0$, установленному в теореме 1.

Заметим, что аналогичное утверждение было ранее установлено Аминовым и Сымом в [5].

Принимая во внимание утверждение 3 и учитывая, что лемма 3 представляет необходимые и достаточные условия для того, чтобы линейная конгруенция была конгруенцией Бьянки, не составляет труда убедиться в истинности следуюшего результата, дополняюшего теорему 1.

Теорема 2. Пусть $F^{2} \subset E^{4}-$ картанова поверхность с гауссовой кривизной $K=-1$. Предположим, что на поверхности $F^{2}$ можнно ввести сопряженнье координаты $u, v$ такие, что семейство координатных линий $v=$ const представлено параллельными геодезическими с ортогональнымми траекториями, т.е. метрика поверхности $F^{2}$ представляется в виде

$$
d s^{2}=(d \varphi(u, v))^{2}+e^{2 \varphi(u, v)}(d v)^{2},
$$

где $\varphi(u, v)$ - некоторая функиия с $\partial_{u} \varphi \neq 0$. Предположим, что сопряженнье координаты $u, v$ нигде не ортогональны, т.е. $\partial_{v} \varphi \neq 0$. Тогда преобразование $F^{2} \rightarrow \widetilde{F}^{2}$, задаваемое формулой

$$
\widetilde{\rho}=\rho-\frac{1}{\partial_{u} \varphi} \partial_{u} \rho
$$

представляет конгруенцию Бъянки. 
Таким образом, картанова поверхность $F^{2}$ в $E^{4}$, допускающая конгруенцию Бьянки, обязана быть псевдосферической и иметь гауссову кривизну $K=-1$. Кроме того, в теоремах 1 и 2 указаны ограничения внутри-геометрического характера, предъявляемые к координатной сети сопряженных линий картановой поверхности $F^{2}$, которые вместе с пседосферичностью являются необходимыми и достаточными условиями существования конгруенции Бьянки. А именно, семейство координатных линий сопряженной сети на $F^{2}$, касательные прямые к которым представляют конгруенцию Бъянки, образовано параллельньми геодезическими с орициклическими ортогональными траекториями. Вообще говоря, произвольная псевдосферическая поверхность в $E^{4}$ не обязана быть картановой и, более того, не обязана нести сопряженную сеть указанного выше специального вида. Поэтому интерес представляет вопрос описания таких поверхностей. Мы дадим ответ на этот вопрос, воспользовавшись уравнениями Гаусса-Кодащии-Риччи, и существенно усилим теоремы 1 и 2.

Заменой $d \widehat{v}=f(v) d v$ всегда можно добиться того, что $f \equiv 1$; не уменьшая общности, будем предполагать, что $v$ специализировано именно таким образом. Еше раз отметим, что $g_{11} g_{22}-g_{12}^{2}=\left(\partial_{u} \varphi\right)^{2} e^{2 \varphi}$, т.е. условие регулярности $F^{2}$ эквивалентно тому, что $\partial_{u} \varphi$ не обращается в нуль. Кроме того, в рассматриваемой ситуации $\partial_{v} \Gamma_{12}^{2}-\Gamma_{12}^{2} \Gamma_{12}^{1}=\partial_{u} \varphi \partial_{v} \varphi$, поэтому условие $(11)$, необходимое для существования конгруенции Бьянки (и эквивалентное регулярности преобразованной поверхности $\widetilde{F}^{2}$ ), будет выполнено тогда и только тогда, когда $\partial_{v} \varphi$ не обрашается в нуль. Мы всегда можем выбрать параметр $v$, сделав при необходимости замену $v \mapsto-v$, таким образом, чтобы производная $\partial_{v} \varphi$ (т.е. коэффициент $g_{12}$ ) была положительной.

Далее, специализируем выбор нормальных векторов $n_{1}, n_{2}$ на $F^{2}$ таким образом, чтобы добиться вьполнения равенства $L_{11}^{2} \equiv 0$. Это всегда можно сделать, направив вектор $n_{1}$ вдоль вектора нормальной кривизны геодезической линии $v=$ const. Вектор $n_{2}$ выберем так, чтобы $n_{1}$ и $n_{2}$ образовывали положительно ориентированный базис нормальной плоскости, а векторы $\partial_{u} \rho, \partial_{v} \rho, n_{1}$ и $n_{2}$ - положительно ориентирванньй базис в $E^{4}$.

После такого выбора сопряженных координат $u, v$ и нормальных векторов $n_{1}, n_{2}$ на рассматриваемой псевдосферической картановой поверхности $F^{2}$ уравнение Гаусса и два из четырех уравнений Кодащи для $F^{2}$ (вытекающие из равенства $\left.\partial_{u u v} \rho=\partial_{v u u} \rho\right)$ будут иметь следующий вид:

$$
\begin{gathered}
L_{11}^{1} L_{22}^{1}+\left(\partial_{u} \varphi\right)^{2} e^{2 \varphi}=0, \\
\partial_{v}\left(\frac{L_{11}^{1} e^{\varphi}}{\partial_{u} \varphi}\right)=0, \\
L_{11}^{1} \mu_{2}=0 .
\end{gathered}
$$

Здесь и в дальнейшем используются обозначения $\mu_{1}=\left\langle\partial_{u} n_{1}, n_{2}\right\rangle, \mu_{2}=\left\langle\partial_{v} n_{1}, n_{2}\right\rangle$. Из (19.2) следует, что функция $Z=L_{11}^{1} e^{\varphi} / \partial_{u} \varphi$ не зависит от $v$. Не составляет труда убедиться в том, что заменой $d \widehat{u}=Z(u) d u$ можно добиться того, чтобы $Z \equiv 1$ (если $Z$ отрицательна, то следует сначала заменить $n_{1}$ на $-n_{1}$ ). Не уменьшая обшности, мы будем предполагать, что именно такие специализация координаты $u$ и выбор нормального вектора $n_{1}$ были сделаны с самого начала, т.е. $L_{11}^{1}=\partial_{u} \varphi e^{-\varphi}$. 
Далее, поскольку точечная коразмерность $F^{2}$ равна 2 , то коэффициент $L_{11}^{1}$ не обрашается в нуль, поэтому из (19.3) получаем $\mu_{2} \equiv 0$. Наконец, из уравнения Гаусса (19.1) следует $L_{22}^{1}=-\partial_{u} \varphi e^{3 \varphi}$.

Таким образом, метрика, вторые квадратичные формы и коэффициенты кручения рассматриваемой поверхности $F^{2} \subset E^{4}$ представляются в виде

$$
\begin{gathered}
g=\left(\begin{array}{cc}
\left(\partial_{u} \varphi\right)^{2} & \partial_{u} \varphi \partial_{v} \varphi \\
\partial_{u} \varphi \partial_{v} \varphi & \left(\partial_{v} \varphi\right)^{2}+e^{2 \varphi}
\end{array}\right), \\
L^{1}=\left(\begin{array}{cc}
\partial_{u} \varphi e^{-\varphi} & 0 \\
0 & -\partial_{u} \varphi e^{3 \varphi}
\end{array}\right), \quad L^{2}=\left(\begin{array}{cc}
0 & 0 \\
0 & L_{22}^{2}
\end{array}\right), \\
\mu_{1}=\mu_{1}, \quad \mu_{2} \equiv 0 .
\end{gathered}
$$

Следует отметить, что теперь сопряженные координаты $u, v$ на $F^{2}$ фиксированы однозначно с точностью до постоянных слагаемых, поскольку выше мы воспользовались шкалирующими заменами $\widehat{u}=\widehat{u}(u), \widehat{v}=\widehat{v}(v)$, чтобы избавиться от функций $f(v)$ и $Z(u)$. Кроме того, однозначно определены и нормальные векторные поля $n_{1}$ и $n_{2}$. С другой стороны, функция $\varphi$ с положительными производными $\partial_{u} \varphi, \partial_{v} \varphi$, которая используется в выражении (20.1) для коэффициентов метрики $F^{2}$, определена однозначно с точностью до постоянного слагаемого. Поэтому если вместо $\varphi$ мы возьмем $\bar{\varphi}=\varphi+c_{0}$, то это повлечет за собой шкалируюшую замену кординат $\bar{u}=e^{-c_{0}} u, \bar{v}=e^{c_{0}} v$. Благодаря такой замене координат мы снова получим выражения вида (20.1)-(20.3) для коэффициентов фундаментальных форм поверхности $F^{2}$.

Найдем теперь коэффициенты фундаментальных форм преобразованной поверхности $\widetilde{F}^{2}$, заданной радиус-вектором (9). Используя выражения (20.1), легко получить $\Gamma_{12}^{2}=\partial_{u} \varphi$, поэтому

$$
\widetilde{\rho}=\rho-\frac{1}{\partial_{u} \varphi} \partial_{u} \rho
$$

Дифференцируя (21), находим касательные векторы $\widetilde{F}^{2}$ :

$$
\partial_{u} \widetilde{\rho}=\partial_{u} \rho-e^{-\varphi} n_{1}, \quad \partial_{v} \widetilde{\rho}=\frac{\partial_{v} \varphi}{\partial_{u} \varphi} \partial_{u} \rho
$$

Еще раз убеждаемся, что $\widetilde{F}^{2}$ будет регулярной тогда и только тогда, когда $\partial_{v} \varphi \neq 0$. Далее, легко проверить, что векторы

$$
\widetilde{n}_{1}=\frac{1}{e^{\varphi} \partial_{u} \varphi}\left(\partial_{v} \varphi \partial_{u} \rho-\partial_{u} \varphi \partial_{v} \rho\right), \quad \widetilde{n}_{2}=-n_{2}
$$

ортогональны касательным векторам $\partial_{u} \widetilde{\rho}, \partial_{v} \widetilde{\rho}$ и образуют ортонормированный базис нормальной плоскости $N_{\widetilde{P}} \widetilde{F}^{2}$. Наконец, вычисляя коэффициенты $\widetilde{g}_{i j}$ метри- 
ки $\widetilde{F}^{2}$, коэффициенты $\widetilde{L}_{i j}^{\sigma}$ вторых фундаментальных форм и коэффициенты кручения $\widetilde{\mu}_{i}$ поверхности $\widetilde{F}^{2}$ относительно нормалей $\widetilde{n}_{\sigma}$ из $(23)$, получаем

$$
\begin{gathered}
\widetilde{g}=\left(\begin{array}{cc}
\left(\partial_{u} \varphi\right)^{2}+e^{-2 \varphi} & \partial_{u} \varphi \partial_{v} \varphi \\
\partial_{u} \varphi \partial_{v} \varphi & \left(\partial_{v} \varphi\right)^{2}
\end{array}\right), \\
\widetilde{L}^{1}=\left(\begin{array}{cc}
\partial_{v} \varphi e^{-3 \varphi} & 0 \\
0 & -\partial_{v} \varphi e^{\varphi}
\end{array}\right), \quad \widetilde{L}^{2}=\left(\begin{array}{cc}
e^{-\varphi} \mu_{1} & 0 \\
0 & 0
\end{array}\right), \\
\widetilde{\mu}_{1}=\left(\partial_{u} \widetilde{n}_{1}, \widetilde{n}_{2}\right)=0, \quad \widetilde{\mu}_{2}=\left(\partial_{v} \widetilde{n}_{1}, \widetilde{n}_{2}\right)=L_{22}^{2} e^{-\varphi} .
\end{gathered}
$$

Из (24.1) сразу же вытекает, что $\widetilde{F}^{2}$, как и $F^{2}$, является псевдосферической поверхностью с постоянной гауссовой кривизной $\widetilde{K}=-1$, а координатные линии $u=$ const на $\widetilde{F}^{2}$ являются параллельньми геодезическими с орициклическими ортогональньми траекториями.

Элементарньм следствием выражений (24.2) является отмеченная вьше сопряженность координатных $u$ - и $v$-линий на $\widetilde{F}^{2}$. Кроме того, из (24.2) следует, что точечная коразмерность $\widetilde{F}^{2}$ равна рангу матрицы

$$
\left(\begin{array}{ccc}
\widetilde{L}_{11}^{1} & \widetilde{L}_{12}^{1} & \widetilde{L}_{22}^{1} \\
\widetilde{L}_{11}^{2} & \widetilde{L}_{12}^{2} & \widetilde{L}_{22}^{2}
\end{array}\right)=\left(\begin{array}{ccc}
\partial_{u} \varphi e^{-3 \varphi} & 0 & -\partial_{v} \varphi e^{\varphi} \\
-e^{-\varphi} \mu_{1} & 0 & 0
\end{array}\right)
$$

Поэтому точечная коразмерность $\widetilde{F}^{2}$ в точке $\widetilde{P}$ будет менњше 2 тогда и только тогда, когда $\mu_{1}$ обратится в нуль в соответствующей точке $P$ на $F^{2}$.

В итоге, мы можем сформулировать утверждение, представляющее один из основных результатов статьи.

Теорема 3. Пусть $F^{2} \subset E^{4}$ - картанова поверхность, $\psi: F^{2} \rightarrow \widetilde{F}^{2}-$ конгруенция Бъянки. Тогда справедливо следующее.

1) Существуют система сопряэсенных координат $u, v$ на $F^{2}$, однозначно определенные нормальные векторные поля $n_{1}, n_{2}$, образующие положстельно ориентированный ортонормированный базис нормальной плоскости $F^{2}$, и функиия $\varphi(u, v)$ с положительными производными $\partial_{u} \varphi>0, \partial_{v} \varphi>0$ такие, что имеют место выражсения (20) для коэффичиентов фундаментальньи форм $F^{2}$. В частности, $F^{2}$ имеет гауссову кривизну $K=-1$, а координатные линии $v=$ const сопряженной сети являются параллельными геодезическими с орициклическими ортогональными траекториями. Функиия $\varphi$ и координаты $и$ и $v$ определены однозначно с точностью до преобразования $\varphi \mapsto \varphi+c_{0}, \quad u \mapsto u e^{-c_{0}}+u_{0}, \quad v \mapsto v e^{c_{0}}+v_{0}$, дде $c_{0}, u_{0}, v_{0}-$ константы.

2) Конгруениия Бвянки $\psi$ задается в виде

$$
\tilde{\rho}=\rho-\frac{1}{\partial_{u} \varphi} \partial_{u} \rho
$$

где $\rho(u, v)$ и $\widetilde{\rho}(u, v)$ - радиус-векторы поверхностей $F^{2}$ и $\widetilde{F}^{2}$.

3) Для коэффичиентов фундаментальных форм поверхности $\widetilde{F}^{2}$ относительно координат $u, v$ и нормальных векторных полей (23) имеют место 
вырахсения (24). В частности, поверхность $\widetilde{F}^{2}$ имеет гауссову кривизну $\widetilde{K}=-1$, координатные $u$ - и v-линии на $\widetilde{F}^{2}$ образуют сопряжсенную сеть, при этом координатные линии $и=$ const являются параллельными геодезическими с орициклическими ортогональными траекториями. Кроме того, $\widetilde{F}^{2}$ является картановой поверхностью тогда и только тогда, когда $\mu_{1} \neq 0$ во всех точках на $F^{2}$.

Таким образом, из существования сопряженной сети на $F^{2}$, у которой одно семейство линий образовано параллельньми геодезическими с орициклическими ортогональными траекториями, вытекает возможность привести фундаментальные формы $F^{2}$ к специальному виду (20). Обратно, если фундаментальные формы произвольной поверхности в $E^{4}$ приводятся к виду (20), то эта поверхность, очевидно, будет картановой псевдосферической поверхностью, несущей сопряженную сеть указанного специального вида. По теореме 2 такая поверхность допускает конгруенцию Бьянки.

ТЕОРемА 4. Предположим, что на псевдосферической картановой поверхности $F^{2} \subset E^{4}$ можсно ввести сопряжсенние кординаты $и, v$ и ортонормированные нормальные векторные поля $n_{1}, n_{2}$ так, что имеют место выражения (20) для коэффициентов фундаментальных форм $F^{2}$, причем $\varphi(u, v)$ имеет положстельные производные $\partial_{u} \varphi>0, \partial_{v} \varphi>0$. Тогда радиус-вектор

$$
\widetilde{\rho}=\rho-\frac{1}{\partial_{u} \varphi} \partial_{u} \rho
$$

определяет преобразование $\psi: F^{2} \rightarrow \widetilde{F}^{2}$, являющееся конгруенцией Бъянки.

Теперь, опираясь на классическую теорему Бонне, мы можем использовать (20) для доказательства существования, описания и построения конкретных примеров псевдосферических картановых поверхностей в $E^{4}$, допускающих конгруенцию Бьянки.

Коэффициенты $L_{22}^{1}, \mu_{1}$ и функция $\varphi$, присутствующие в формулах $(20)$, не могут быть произвольными. Они обязаны удовлетворять некоторым соотношениям, являющимимся следствием уравнений Гаусса-Кодацци-Риччи.

Для удобства записи вместо коэффициентов $L_{22}^{2}$ и $\mu_{1}$ мы введем в рассмотрение функции $P(u, v), Q(u, v)$, положив $L_{22}^{2}=e^{\varphi} P(u, v), \mu_{1}=Q(u, v)$. Тогда выражения (20) перепишутся следующим образом:

$$
\begin{gathered}
g=\left(\begin{array}{cc}
\left(\partial_{u} \varphi\right)^{2} & \partial_{u} \varphi \partial_{v} \varphi \\
\partial_{u} \varphi \partial_{v} \varphi & \left(\partial_{v} \varphi\right)^{2}+e^{2 \varphi}
\end{array}\right) \\
L^{1}=\left(\begin{array}{cc}
\partial_{u} \varphi e^{-\varphi} & 0 \\
0 & -\partial_{u} \varphi e^{3 \varphi}
\end{array}\right), \quad L^{2}=\left(\begin{array}{cc}
0 & 0 \\
0 & e^{\varphi} P(u, v)
\end{array}\right), \\
\mu_{1}=Q(u, v), \quad \mu_{2} \equiv 0 .
\end{gathered}
$$

Часть уравнений (уравнение Гаусса и два из четырех уравнений Кодацци) уже выполнены, они использовались при получении выражений (20). Легко проверить, 
что оставшиеся два уравнения Кодацщи и уравнение Риччи для $F^{2}$ сводятся к трем следуюшим уравнениям для трех функций $\varphi(u, v), P(u, v), Q(u, v)$ :

$$
\begin{gathered}
\partial_{u u}^{2} \varphi \cdot e^{2 \varphi}+2\left(\partial_{u} \varphi\right)^{2} e^{2 \varphi}-\partial_{v v}^{2} \varphi \cdot e^{-2 \varphi}+2\left(\partial_{v} \varphi\right)^{2} e^{-2 \varphi}+P Q+1=0, \\
\partial_{u} P-\partial_{u} \varphi Q e^{2 \varphi}=0 \\
\partial_{v} Q+\partial_{v} \varphi P e^{-2 \varphi}=0
\end{gathered}
$$

при этом $\partial_{u} \varphi>0$ (регулярность $\left.F^{2}\right), \partial_{v} \varphi>0$ (сопряженная сеть на $F^{2}$ нигде не ортогональна, эквивалентно - регулярность $\widetilde{F}^{2}$ ), $P \neq 0$ (точечная коразмерность $F^{2}$ равна 2$), Q \neq 0$ (точечная коразмерность $\widetilde{F}^{2}$ равна 2 ).

Обратно, любому решению $\varphi(u, v), P(u, v), Q(u, v)$ системы уравнений $(26)$ с $\partial_{u} \varphi>0, \partial_{v} \varphi>0, P \neq 0$ будет соответствовать по классической теореме Бонне псевдосферическая картанова поверхность, чьи фундаментальные формы выражаются в терминах $\varphi(u, v), P(u, v), Q(u, v)$ с помощью формул (25). Как следствие такая поверхность будет нести сопряженную сеть, у которой одно семейство линий представлено параллельньми геодезическими с орициклическими ортогональными траекториями.

Таким образом, мы можем утверждать, что псевдосферическая картанова поверхность $F^{2}$ в $E^{4}$, несущая конгруенцию Бьянки, посредством формул (25) описывается решением $\varphi(u, v), P(u, v), Q(u, v)$ системы нелинейных дифференциальных уравнений (26), удовлетворяющих условиям $\partial_{u} \varphi>0, \partial_{v} \varphi>0, P \neq 0$. При этом преобразованная поверхность $\widetilde{F}^{2}$ будет картановой тогда и только тогда, когда выполнено еще и условие $Q \neq 0$. Такое описание однозначно с точностью до преобразования переменных $u \mapsto u e^{-c_{0}}+u_{0}, v \mapsto v e^{c_{0}}+v_{0}$ и функций $\varphi \mapsto \varphi+c_{0}$, $P \mapsto P e^{-c_{0}}, Q \mapsto Q e^{c_{0}}$, где $c_{0}, u_{0}, v_{0}$ - константы; легко проверить, что система уравнений (26) действительно инварианта относительно таких преобразований.

Теперь запишем выражения (24) для коэффициентов фундаментальных форм преобразованной поверхности $\widetilde{F}^{2}$ в терминах $\varphi(u, v), P(u, v), Q(u, v)$ :

$$
\begin{gathered}
\widetilde{g}=\left(\begin{array}{cc}
\left(\partial_{u} \varphi\right)^{2}+e^{-2 \varphi} & \partial_{u} \varphi \partial_{v} \varphi \\
\partial_{u} \varphi \partial_{v} \varphi & \left(\partial_{v} \varphi\right)^{2}
\end{array}\right), \\
\widetilde{L}^{1}=\left(\begin{array}{cc}
\partial_{v} \varphi e^{-3 \varphi} & 0 \\
0 & -\partial_{v} \varphi e^{\varphi}
\end{array}\right), \\
\widetilde{L}^{2}=\left(\begin{array}{cc}
e^{-\varphi} Q & 0 \\
0 & 0
\end{array}\right), \\
\widetilde{\mu}_{1}=\left(\partial_{u} \widetilde{n}_{1}, \widetilde{n}_{2}\right)=0, \quad \widetilde{\mu}_{2}=\left(\partial_{v} \widetilde{n}_{1}, \widetilde{n}_{2}\right)=P .
\end{gathered}
$$

Легко видеть, что если произвести замену $\widetilde{u}=-v, \widetilde{v}=-u$ и ввести функции $\widetilde{P}(\widetilde{u}, \widetilde{v})=-Q(u, v), \widetilde{Q}(\widetilde{u}, \widetilde{v})=P(u, v), \widetilde{\varphi}(\widetilde{u}, \widetilde{v})=-\varphi(u, v)$, то выражения $(27)$ примут точно такую же форму, что и выражения (25). Иначе говоря, преобразованная поверхность $\widetilde{F}^{2}$ представляется функциями $-\varphi(-v,-u),-Q(-v,-u)$, $-P(-v,-u)$.

Таким образом, преобразование псевдосферических картановъх поверхностей в $E^{4}$ посредством конгруенции Бьянки соответствует алгебраическому инволютивному преобразованию решений

$$
\{\varphi(u, v), P(u, v), Q(u, v)\} \mapsto\{-\varphi(-v,-u),-Q(-v,-u),-P(-v,-u)\}
$$


системь дифференциальных уравнений (26), которое тоже естественно называть преобразованием Бьянки.

Отдельный интерес представляет вопрос о разрешимости системы уравнений (26). Мы рассмотрим два частных случая, в которых удается упростить задачу и даже получить конкретные решения.

ПримеР 1. Найдем решение системы (26) вида “бегушей волны":

$$
\varphi=f(a u+b v), \quad P=p(a u+b v), \quad Q=q(a u+b v) .
$$

Подставляя (29) в (26), получаем систему трех обыкновенных дифференциальных уравнений:

$$
\begin{gathered}
p^{\prime}-f^{\prime} q e^{2 f}=0, \\
q^{\prime}+f^{\prime} p e^{-2 f}=0 \\
\left(\frac{a^{2} e^{2 f}+b^{2} e^{-2 f}}{2}\right)^{\prime \prime}+p q+1=0
\end{gathered}
$$

для трех функций $p(\tau), q(\tau), f(\tau)$ одной переменной $\tau=a u+b v$.

Из уравнений (30.1), (30.2) вытекает, что

$$
p e^{-f}-q e^{f}=\text { const. }
$$

Это уравнение разрешается следующим образом:

$$
p=e^{f}\left(\xi+c_{1}\right), \quad q=e^{-f}\left(\xi-c_{1}\right)
$$

где $\xi=\xi(\tau), c_{1}=$ const. Подставляя $(31)$ в $(30.1),(30.2)$, получаем

$$
\xi^{\prime}+2 c_{1} f^{\prime}=0
$$

Проинтегрируем данное уравнение и подставим решение $\xi=c_{2}-2 c_{1} f$ в (31):

$$
p=e^{f}\left(c_{2}+c_{1}-2 c_{1} f\right), \quad q=e^{-f}\left(c_{2}-c_{1}-2 c_{1} f\right),
$$

здесь $c_{2}$ - произвольная константа. Уравнение (30.3) для $f$ теперь принимает следуюшую форму:

$$
\left(\frac{a^{2} e^{2 f}+b^{2} e^{-2 f}}{2}\right)^{\prime \prime}+\left(c_{2}-2 c_{1} f\right)^{2}-c_{1}^{2}+1=0
$$

В частности, если $c_{1}=0$, то получаем уравнение

$$
\left(\frac{a^{2} e^{2 f}+b^{2} e^{-2 f}}{2}\right)^{\prime \prime}+c_{2}^{2}+1=0
$$


которое легко проинтегрировать:

$$
\frac{a^{2} e^{2 f}+b^{2} e^{-2 f}}{2}=-C_{1}\left(\tau+C_{2}\right)^{2}+C_{3}
$$

здесь $C_{1}=\left(c_{2}^{2}+1\right) / 2$, а $C_{2}, C_{3}$ - произвольные константы. Находя $f$ и подставляя в $(32)$, получаем явные выражения для $p$ и $q$. Как следствие из (29) получаем конкретное решение типа “бегущей волны” системы уравнений (26). Преобразование Бьянки переводит построенное решение $\varphi=f(a u+b v), P=p(a u+b v)$, $Q=q(a u+b v)$ системы $(26)$ в новое решение $\varphi=-f(-b u-a v), P=q(-b u-a v)$, $Q=p(-b u-a v)$ той же системы.

$\mathrm{B}$ самом простом случае, при $a=1, b=1, c_{2}=1, C_{2}=0$ и $C_{3}=2$, получаем решение

$$
\begin{aligned}
& \varphi(u, v)=\frac{1}{2} \ln \left(2-(u+v)^{2}+\sqrt{\left(2-(u+v)^{2}\right)^{2}-1}\right), \\
& P(u, v)=\sqrt{2-(u+v)^{2}+\sqrt{\left(2-(u+v)^{2}\right)^{2}-1}} \\
& Q(u, v)=\sqrt{2-(u+v)^{2}-\sqrt{\left(2-(u+v)^{2}\right)^{2}-1}}
\end{aligned}
$$

определенное в полосе $|u+v|<1$. С помощью преобразования Бьянки (28) получаем другое решение системы (26):

$$
\begin{aligned}
& \varphi(u, v)=-\frac{1}{2} \ln \left(2-(u+v)^{2}+\sqrt{\left(2-(u+v)^{2}\right)^{2}-1}\right) \\
& P(u, v)=-\sqrt{2-(u+v)^{2}-\sqrt{\left(2-(u+v)^{2}\right)^{2}-1}} \\
& Q(u, v)=-\sqrt{2-(u+v)^{2}+\sqrt{\left(2-(u+v)^{2}\right)^{2}-1}} .
\end{aligned}
$$

ПРимеР 2. Найдем решение системы уравнений (26) в виде

$$
\varphi=\varphi(u, v), \quad P=\mathscr{P}(\varphi(u, v)), \quad Q=\mathscr{Q}(\varphi(u, v))
$$

Уравнения (26.2), (26.3) перепишутся так:

$$
\mathscr{P}_{\varphi}^{\prime}-\mathscr{Q} e^{2 \varphi}=0, \quad \mathscr{Q}_{\varphi}^{\prime}+\mathscr{P} e^{-2 \varphi}=0
$$

откуда находим

$$
\mathscr{P}=e^{\varphi}\left(c_{1} \varphi+c_{2}\right), \quad \mathscr{Q}=e^{-\varphi}\left(c_{1} \varphi+c_{1}+c_{2}\right),
$$

где $c_{1}$ и $c_{2}$ - произвольные константы. Оставшееся уравнение $(26.1)$ для $\varphi$ примет вид

$$
\partial_{u u}^{2} e^{2 \varphi}+\partial_{v v}^{2} e^{-2 \varphi}+2\left(\left(c_{1} \varphi+c_{2}\right)^{2}+c_{1}\left(c_{1} \varphi+c_{2}\right)+1\right)=0
$$


В простейшем случае при $c_{1}=0$ получаем уравнение

$$
\partial_{u u}^{2} e^{2 \varphi}+\partial_{v v}^{2} e^{-2 \varphi}+C=0
$$

где $C=2\left(c_{2}^{2}+1\right)$ - константа. Легко видеть, что вместе с $\varphi(u, v)$ решением этого уравнения будет и $-\varphi(-u,-v)$.

В завершение статьи сформулируем ряд вопросов, представляюших с нашей точки зрения определенный интерес в связи с дальнейшим исследованием преобразований Бьянки поверхностей в $E^{4}$.

1) Предположим, что первое преобразование Лапласа псевдосферической картановой поверхности $F^{2}$ в $E^{4}$ представляет конгруенцию Бьянки. Что можно сказать о минус первом преобразовании Лапласа поверхности $F^{2}$ в $E^{4}$ ? Будет ли оно тоже представлять конгруенцию Бьянки?

2) Сушествует ли псевдосферическая картанова поверхность $F^{2}$ в $E^{4}$, первое и минус первое преобразования Лапласа которой представляют конгруенции Бьянки? Иначе говоря, сушествует ли псевдосферическая картанова поверхность $F^{2}$ в $E^{4}$, у которой каждое из двух семейств линий сопряженной сети представляется параллельньми геодезическими с ортогональными траекториями? Если ответ положителен, то будут ли в свою очередь обладать таким же свойством и преобразованные поверхности?

\section{Приложение}

Выясним геометрический смысл инвариантных выражений, стоящих в правых частях в формулах (13) и (14) для угла между касательньпи плоскостями и расстояния между соответствующими точками линейной конгруенции $F^{2} \rightarrow \widetilde{F}^{2}$. Для этого запишем геодезическую кривизну $k_{g}$ и нормальную кривизну $k_{n}$ (длину вектора нормальной кривизны) координатной линии $v=$ const сопряженной сети на $F^{2}$ :

$$
k_{g}=\frac{\Gamma_{11}^{2}}{{\sqrt{g_{11}}}^{3}} \sqrt{g_{11} g_{22}-\left(g_{12}\right)^{2}}, \quad k_{n}=\frac{\sqrt{\left(L_{11}^{1}\right)^{2}+\left(L_{11}^{2}\right)^{2}}}{g_{11}} .
$$

Формулу (14) для угла $\omega$ мы можем теперь переписать следующим образом:

$$
\cos \omega=\frac{k_{g}}{\sqrt{k_{g}^{2}+k_{n}^{2}}}=\frac{k_{g}}{k},
$$

где $k$ - кривизна линии $v=\mathrm{const}$, рассматриваемой как кривая в $E^{4}$. Как следствие условие $\mathrm{B}_{2}$ ) в определении конгруенции Бьянки будет вьполнено, т.е. $\cos \omega=0$, тогда и только тогда, ког да координатные линии $v=$ const сопряженной сети на $F^{2}$ являются геодезическими.

Аналогичньм образом можно переписать и формулу (13) для длины $l$ между соответствующими точками линейной конгруенции:

$$
l=\frac{1}{\left|k_{g}^{\perp}+k_{g} \operatorname{ctg} \theta\right|},
$$


где $k_{g}^{\perp}-$ геодезическая кривизна ортогональных траекторий линий $v=$ const, а $\theta$ - угол между координатными линиями сопряженной сети. Если $k_{g}=0$, то $l=1 /\left|k_{g}^{\perp}\right|$, поэтому условие $\left.\mathrm{B}_{1}\right)$ в определении конгруенции Бьянки будет выполнено, т.е. $l=1$, тогда и только тогда, когда $\left|k_{g}^{\perp}\right|=1$. Таким образом, геодезическая кривизна ортогональных траекторий координатных линий $v=$ const на $F^{2}$ постоянна и равна 1 . Как результат мы получаем семейство геодезических линий с ортогональными траекториями постоянной геодезической кривизны 1 . Такая ситуация возможна лишь на плоскости Лобачевского, когда речь идет о параллельных геодезических с ортогональными орициклическими траекториями.

\section{Список литературы}

1. Аминов Ю. А. Геометрия подмногообразий. Киев: Наукова думка, 2000.

2. Tenenblat $K$. Transformations of manifolds and applications to differential equations. London: Longman, 1998.

3. Tenenblat K., Terng C.-L. Backlund theorem for $n$-dimensional submanifolds of $R^{2 n-1}$ // Ann. of Math. (2). 1980. V. 111. P. 477-490.

4. Terng C.-L. A higher dimension generalisation of the Sine-Gordon equation and its soliton theory // Ann. of Math. (2). 1980. V. 111. P. 491-510.

5. Aminov Yu., Sym A. On Bianchi and Backlund transformations of two-dimensional surfaces in $E^{4}$ // Math. Phys. Anal. Geom. 2000. V. 3. № 1. P. 75-89.

6. Eisenhart L.P. A treatise on the differential geometry of curves and surfaces. New York: Ginn, 1909

7. Шефель С. З. Геометрические свойства погруженных многообразий // Сиб. матем. журн. 1985. Т. 26. №1. С. 170-188.

8. Борисенко А. А., Николаевский Ю. А. Многообразия Грассмана и грассманов образ подмногообразий // УМН. 1991. Т. 46. № 2. С. 41-83.

E-mail: gorkaviy@ilt.kharkov.ua 